\title{
On a formula of Pollaczek and Spitzer \\ by
}

LAJOS TAKÁCS* (Cleveland, Ohio)

Abstract. In 1952 F. Pollaczek and in 1956 F. Spitzer found a formula which makes it possible to determine the distribution of the maximal partial sum of $n$ mutually independent and identically distributed real random variables. This paper contains two simple and elementary proofs and a generalization of the formula of Pollaczek and Spitzer.

1. Introduction. Let $\xi_{1}, \xi_{2}, \ldots, \xi_{n}, \ldots$ be mutually independent and identically distributed real random variables and write

$$
\boldsymbol{E}\left\{e^{-s \xi_{n}}\right\}=\varphi(s)
$$

for $\operatorname{Re}(s)=0$. Define $\zeta_{0}=0, \zeta_{n}=\xi_{1}+\ldots+\xi_{n}$ for $n=1,2, \ldots, \zeta_{n}^{+}$ $=\max \left(0, \zeta_{n}\right)$ for $n=0,1,2, \ldots$, and $\eta_{n}^{*}=\max \left(\zeta_{0}, \zeta_{1}, \ldots, \zeta_{n}\right)$ for $n=0,1,2, \ldots$ Introduce the Laplace-Stieltjes transforms

$$
\begin{aligned}
& \text { (2) } \quad \boldsymbol{E}\left\{e^{-s \xi_{n}}\right\}=\varphi_{n}(s) \text { for } \operatorname{Re}(s)=0 \text { and } n=0,1,2, \ldots \text {, } \\
& \text { (3) } \boldsymbol{E}\left\{e^{-s \zeta_{n}^{+}}\right\}=\varphi_{n}^{+}(s) \text { for } \operatorname{Re}(s) \geqslant 0 \text { and } n=0,1,2, \ldots \text {, } \\
& \text { and } \\
& \text { (4) } \quad \boldsymbol{E}\left\{e^{-s n_{n}^{*}}\right\}=\Phi_{n}(s) \quad \text { for } \quad \operatorname{Re}(s) \geqslant 0 \text { and } n=0,1,2, \ldots
\end{aligned}
$$

In 1952 F. Pollaczek [1] and in 1956 F. Spitzer [2] proved that if $\operatorname{Re}(s) \geqslant 0$ and $|\varrho|<1$, then

$$
\sum_{n=0}^{\infty} \Phi_{n}(s) \varrho^{n}=\exp \left\{\sum_{k=1}^{\infty} \frac{\varrho^{k}}{k} \varphi_{k}^{+}(s)\right\} .
$$

In this paper we shall give two simple and elementary proofs for (5) and we shall also prove a generalization of (5).

2. Auxiliary theorems. It will be convenient to introduce an operator $\boldsymbol{A}$. Let us suppose that $\xi$ is a real random variable with distribution function $\boldsymbol{P}\{\xi \leqslant x\}=F(x)$ and write $\xi^{+}=\max (0, \xi)$. Then

$$
\varphi(s)=\boldsymbol{E}\left\{e^{-s \xi}\right\}=\int_{-\infty}^{\infty} e^{-s x} d F(x)
$$

* This research was supported by the National Science Foundation under Contract No. GP-7847. 
exists for $\operatorname{Re}(s)=0$ and determines uniquely

(7) $\quad \varphi^{+}(s)=\boldsymbol{E}\left\{e^{-s \xi^{+}}\right\}=F(0)+\int_{+0}^{\infty} e^{-s x} d F(x)$ for $\operatorname{Re}(s) \geqslant 0$.

We shall write

If

$$
\varphi^{+}(s)=A \varphi(s) \quad \text { for } \quad \operatorname{Re}(s) \geqslant 0 .
$$

$$
\gamma(s)=c_{1} \varphi_{1}(s)+\ldots+c_{n} \varphi_{n}(s),
$$

where $n$ is a positive integer, $c_{1}, c_{2}, \ldots, c_{n}$ are complex numbers and $\varphi_{1}(s), \varphi_{2}(s), \ldots, \varphi_{n}(s)$ are Laplace-Stieltjes transforms of real random variables, then let us define

(10) $\quad \boldsymbol{A} \gamma(s)=c_{1} \boldsymbol{A}_{\varphi_{1}}(s)+\ldots+c_{n} \boldsymbol{A}_{p_{n}}(s)$ for $\operatorname{Re}(s) \geqslant 0$.

LEMMA 1. If $\gamma_{1}(s)$ and $\gamma_{2}(s)$ are of the form (9), then we have

(11) $\boldsymbol{A}\left\{\gamma_{1}(s) \boldsymbol{A} \gamma_{2}(s)\right\}+\boldsymbol{A}\left\{\gamma_{2}(s) \boldsymbol{A}_{\gamma_{1}}(s)\right\}=\boldsymbol{A}\left\{\gamma_{1}(s) \gamma_{2}(s)\right\}+\left(\boldsymbol{A} \gamma_{1}(s)\right)\left(\boldsymbol{A} \gamma_{2}(s)\right)$

for $\operatorname{Re}(s) \geqslant 0$.

Proof. First let us suppose that $\gamma_{1}(s)$ and $\gamma_{2}(s)$ are Laplace-Stieltjes transforms of real random variables. We can easily see that for any two real random variables $\chi_{1}$ and $\chi_{2}$ we have

(12) $\boldsymbol{P}\left\{\max \left(0, \chi_{1}, \chi_{1}+\chi_{2}\right) \leqslant x\right\}+\boldsymbol{P}\left\{\max \left(0, \chi_{2}, \chi_{1}+\chi_{2}\right) \leqslant x\right\}$

$$
=\boldsymbol{P}\left\{\max \left(0, \chi_{1}+\chi_{2}\right) \leqslant x\right\}+\boldsymbol{P}\left\{\max \left(0, \chi_{1}\right)+\max \left(0, \chi_{2}\right) \leqslant \infty\right\}
$$

for all $x$. If we suppose that $\chi_{1}$ and $\chi_{2}$ are independent random variables for which $\boldsymbol{E}\left\{e^{-s z_{1}}\right\}=\gamma_{1}(s)$ and $\boldsymbol{E}\left\{e^{-s x_{2}}\right\}=\gamma_{2}(s)$ whenever $\operatorname{Re}(s)=0$, and if we form the Laplace-Stieltjes transform of (12), then we obtain (11) in this particular case. The general case can be reduced immediately to this particular case by using the representation (9). This completes the proof of the lemma.

Finally we note that if $\boldsymbol{A} \gamma_{1}(s)=\gamma_{1}(s)$ and $\boldsymbol{A} \gamma_{2}(s)=\gamma_{2}(s)$ for $\operatorname{Re}(s)$ $\geqslant 0$, then $\boldsymbol{A}\left\{\gamma_{1}(s) \gamma_{2}(s)\right\}=\gamma_{1}(s) \gamma_{2}(s)$ for $\operatorname{Re}(s) \geqslant 0$. Furthermore, if $\boldsymbol{A} \gamma_{1}(s)=\boldsymbol{A} \gamma_{2}(s)=0$ for $\operatorname{Re}(s) \geqslant 0$, then $\boldsymbol{A}\left\{\gamma_{1}(s) \gamma_{2}(s)\right\}=0$ for $\operatorname{Re}(s) \geqslant 0$.

3. First proof of $(5)$. For $n=1,2, \ldots$ we can write that (13) $\eta_{n}^{*}=\max \left(0, \xi_{1}, \xi_{1}+\xi_{2}, \ldots, \xi_{1}+\ldots+\xi_{n}\right)=\max \left(0, \xi_{1}+\bar{\eta}_{n-1}^{*}\right)$, where $\bar{\eta}_{n-1}^{*}=\max \left(0, \xi_{2}, \xi_{2}+\xi_{3}, \ldots, \xi_{2}+\ldots+\xi_{n}\right)$ has the same distribution as $\eta_{n-1}^{*}$ and is independent of $\xi_{1}$. Hence it follows that for $\operatorname{Re}(s) \geqslant 0$ and $n=1,2, \ldots$ we have

$$
\Phi_{n}(s)=\boldsymbol{A}\left\{\varphi(s) \Phi_{n-1}(s)\right\}
$$

and obviously $\Phi_{0}(s)=1$ for $\operatorname{Re}(s) \geqslant 0$.
Starting from $\Phi_{0}(s)$ we can obtain $\Phi_{n}(s)$ for every $n=1,2, \ldots$ by the recurrence formula (14). However, we can deduce from (14) a simpler recurrence relation. If $\operatorname{Re}(s) \geqslant 0$ and $n=1,2, \ldots$, then we have

$$
\Phi_{n}(s)=\frac{1}{n} \sum_{k=1}^{n} \varphi_{k}^{+}(s) \Phi_{n-k}(s) .
$$

This can be proved by mathematical induction. If $n=1$, then (15) is true. Suppose that (15) is true for $n(n=1,2, \ldots)$. We shall prove that it is true for $n+1$. Hence it follows that (15) is true for every $n=1,2, \ldots$ By (14) and (15) we can write that

$$
\Phi_{n+1}(s)=\boldsymbol{A}\left\{\varphi(s) \Phi_{n}(s)\right\}=\frac{1}{n} \sum_{k=1}^{n} \boldsymbol{A}\left\{\varphi(s) \varphi_{k}^{+}(s) \Phi_{n-y_{k}}(s)\right\}
$$

for $\operatorname{Re}(s) \geqslant 0$. If we apply Lemma 1 to $\gamma_{1}(s)=\varphi(s) \Phi_{n-k}(s)$ and $\gamma_{2}(s)$ $=\varphi_{k}(s)$, then we obtain that

$$
\text { (17) } \begin{aligned}
& \boldsymbol{A}\left\{\varphi(s) \varphi_{k}^{+}(s) \Phi_{n-k}(s)\right\}=\boldsymbol{A}\left\{\varphi_{k+1}(s) \Phi_{n-k}(s)\right\}+ \\
&+\varphi_{k}^{+}(s) \Phi_{n-k+1}(s)-\boldsymbol{A}\left\{\varphi_{k}(s) \Phi_{n-l k+1}(s)\right\} .
\end{aligned}
$$

If we put (17) into (16), then we obtain that

$$
\Phi_{n+1}(s)=\frac{1}{n} \sum_{k=1}^{n+1} \varphi_{k}^{+}(s) \Phi_{n-k+1}(s)-\frac{1}{n} \Phi_{n+1}(s),
$$

that is,

$$
\Phi_{n+1}(s)=\frac{1}{n+1} \sum_{k=1}^{n+1} p_{k}^{+}(s) \Phi_{n-k+1}(s) \quad \text { for } \quad \operatorname{Re}(s) \geqslant 0 .
$$

Hence (15) is true if $n$ is replaced by $n+1$, and this completes the proof of $(15)$.

If we introduce the generating function

$$
U(s, \varrho)=\sum_{n=0}^{\infty} \Phi_{n}(s) \varrho^{n} \quad \text { for } \quad \operatorname{Re}(s) \geqslant 0 \quad \text { and } \quad|\varrho|<1
$$

then by (15) we obtain that

$$
\frac{\partial U(s, \varrho)}{\partial \varrho}=U(s, \varrho) \sum_{k=1}^{\infty} \varphi_{k}^{+}(s) \varrho^{k-1} .
$$

Since $U(s, 0)=1$, it follows that

$$
\log U(s, \varrho)=\sum_{k=1}^{\infty} \frac{\varphi_{k}^{+}(s)}{k} \varrho^{k}
$$

for $\operatorname{Re}(s) \geqslant 0$ and $|\varrho|<1$. This completes the proof of $(5)$. 
4. Second proof of (5). For $n=1,2, \ldots$ let us define the polynomials (23) $Q_{n}\left(x_{1}, x_{2}, \ldots, x_{n}\right)=\sum_{k_{1}+2 k_{2}+\ldots+n k_{n}=n} \frac{1}{k_{1} ! k_{2} ! \ldots k_{n} !}\left(\frac{x_{1}}{1}\right)^{k_{1}}\left(\frac{x_{2}}{2}\right)^{k_{2}} \ldots\left(\frac{x_{n}}{n}\right)^{k_{n}}$, where $k_{1}, k_{2}, \ldots, k_{n}$ are non-negative integers.

LEMMA 2. If $x_{1}, x_{2}, \ldots, x_{n}, \ldots$ are complex numbers for which $\left|x_{n}\right| \leqslant 1$ $(n=1,2, \ldots)$ and $|\varrho|<1$, then

$$
1+\sum_{n=1}^{\infty} Q_{n}\left(x_{1}, x_{2}, \ldots, x_{n}\right) \varrho^{n}=\exp \left\{\sum_{k=1}^{\infty} \frac{\varrho^{l}}{k} x_{l_{l}}\right\} .
$$

Proof. The proof is immediate. If we form the coefficient of $\varrho^{n}$ in the power series expansion of the right-hand side of (24), then we obtain $Q_{n}\left(x_{1}, x_{2}, \ldots, x_{n}\right)$ for $n=1,2, \ldots$

We note that if $|y| \leqslant 1$ and we multiply (24) by

$$
1-\varrho y=\exp \left\{-\sum_{k=1}^{\infty} \frac{\varrho^{k}}{k} y^{k}\right\}
$$

then by forming the coefficient of $\varrho^{n}$ we obtain that

$$
\begin{aligned}
Q_{n}\left(x_{1}, x_{2}, \ldots, x_{n}\right)-y Q_{n-1}\left(x_{1}, x_{2}, \ldots, x_{n-1}\right) \\
=Q_{n}\left(x_{1}-y, x_{2}-y^{2}, \ldots, x_{n}-y^{n}\right)
\end{aligned}
$$

for $n=1,2, \ldots$ and here $Q_{0} \equiv 1$.

Now we shall prove that for $\operatorname{Re}(s) \geqslant 0$ and $n=1,2, \ldots$ we have

$$
\Phi_{n}(s)=Q_{n}\left(\varphi_{1}^{+}(s), \varphi_{2}^{+}(s), \ldots, \varphi_{n}^{+}(s)\right)
$$

and evidently $\Phi_{0}(s)=1$. This implies (5) if we use (24).

Since the right-hand side of (27) is a polynomial of $\varphi_{1}^{+}(s), \varphi_{2}^{+}(s), \ldots$ $\ldots, \varphi_{n}^{+}(s)$ and $\boldsymbol{A} \varphi_{j}^{+}(s)=\varphi_{j}^{+}(s)$ for $\operatorname{Re}(s) \geqslant 0$ and $j=1,2, \ldots, n$, it follows that

$$
\boldsymbol{A} \hat{\Phi}_{n}(s)=\Phi_{n}(s)
$$

On the other hand by (26) we can write down that

(29) $\Phi_{n}(s)-\varphi(s) \Phi_{n-1}(s)$

$$
=Q_{n}\left(\varphi_{1}^{+}(s)-p_{1}(s), p_{2}^{+}(s)-p_{2}(s), \ldots, \varphi_{n}^{+-}(s)-\varphi_{n}(s)\right)
$$

for $n=1,2, \ldots$ and $\operatorname{Re}(s) \geqslant 0$. Since the right-hand side of $(29)$ is a polynomial of $\varphi_{1}^{+}(s)-\varphi_{1}(s), p_{2}^{+}(s)-\varphi_{2}(s), \ldots, \varphi_{n}^{+}(s)-\varphi_{n}(s)$ and $\boldsymbol{A}\left\{p_{j}^{-+}(s)-\right.$ $\left.-\varphi_{j}(s)\right\}=0$ for $\operatorname{Re}(s) \geqslant 0$, it follows that

$$
\text { (30) } \quad \boldsymbol{A}\left\{\Phi_{n}(s)-\varphi(s) \Phi_{n-1}(s)\right\}=0
$$

for $n=1,2, \ldots$ and $\operatorname{Re}(s) \geqslant 0$.
By (28) and (30) we obtain that

$$
\Phi_{n}(s)=\boldsymbol{A}\left\{\varphi(s) \Phi_{n-1}(s)\right\} \quad \text { for } \quad n=1,2, \ldots
$$

This is in agreement with (14) and therefore (27) is indeed correct.

5. A generalization of (5). Let us suppose that $\eta_{0}$ is a non-negative random variable and $\xi_{n}(n=1,2, \ldots)$ is a sequence of mutually independent and identically distributed real random variables. Suppose that $\left\{\xi_{n}\right\}$ and $\eta_{0}$ are also independent. Denote by $\varphi(s)$ the Laplace-Stieltjes transform of $\xi_{n}$.

Let us define a sequence of random variables $\eta_{n}(n=0,1,2, \ldots)$ by the following recurrence relation

$$
\eta_{n}=\max \left(0, \eta_{n-1}+\xi_{n}\right) \quad \text { for } \quad n=1,2, \ldots
$$

If $\boldsymbol{P}\left\{\eta_{0}=0\right\}=1$, then obviously $\boldsymbol{P}\left\{\eta_{n} \leqslant x\right\}=\boldsymbol{P}\left\{\eta_{n}^{*} \leqslant x\right\}$ for $n$ $=1,2, \ldots$, where $\eta_{n}^{*}(n=0,1,2, \ldots)$ is defined in the Introduction. Now we are interested in finding the distribution of $\eta_{n}$ in the general case. Let

(33) $\boldsymbol{E}\left\{e^{-s \eta} n\right\}=\Omega_{n}(s)$ for $\operatorname{Re}(s) \geqslant 0$ and $n=0,1,2, \ldots$

Theoreir 1. For $\operatorname{Re}(s) \geqslant 0$ and $n=1,2, \ldots$ we have

$$
\Omega_{n}(s)=\sum_{k=0}^{n} \Phi_{n-k}(s) \boldsymbol{A}\left\{\Omega_{0}(s) Q_{k}^{*}(s)\right\}
$$

where $\Phi_{k}(s)(k=0,1,2, \ldots, n)$ is given by $(27), Q_{0}^{*}(s)=1$ and

(35) $\quad Q_{k}^{*}(s)=Q_{k}\left(\varphi_{1}(s)-\varphi_{1}^{+}(s), \varphi_{2}(s)-\varphi_{2}^{+}(s), \ldots, \varphi_{k}(s)-\varphi_{k}^{+}(s)\right)$

for $k=1,2, \ldots, n$ and the right-hand side of (35) can be obtained by (23).

Proof. We shall prove that if $\Omega_{n}(s)$ is given by (34), then for $n=$ $=0,1,2, \ldots$

$$
\boldsymbol{A}\left\{\varphi(s) \Omega_{n}(s)\right\}=\Omega_{n+1}(s)
$$

if $\operatorname{Re}(s) \geqslant 0$. Hence it follows that (34) is indeed the correct formula. By (34)

$$
\boldsymbol{A}\left\{\varphi(s) \Omega_{n}(s)\right\}=\sum_{k=0}^{n} \boldsymbol{A}\left\{\varphi(s) \Phi_{n-k}(s) \boldsymbol{A}\left\{\Omega_{0}(s) Q_{k}^{*}(s)\right\}\right\} .
$$

If we apply Lemma 1 to the functions $\gamma_{1}(s)=\varphi(s) \Phi_{n-k}(s)$ and $\gamma_{2}(s)$ $=\Omega_{0}(s) Q_{k}^{*}(s)$, then we obtain that

(38) $\quad \boldsymbol{A}\left\{\varphi(s) \Phi_{n-k}(s) \boldsymbol{A}\left\{\Omega_{0}(s) Q_{k}^{*}(s)\right\}\right\}=\boldsymbol{A}\left\{\varphi(s) \Phi_{n-k}(s) \Omega_{0}(s) Q_{k}^{*}(s)\right\}+$ $+\Phi_{n-k+1}(s) \boldsymbol{A}\left\{\Omega_{0}(s) Q_{k}^{*}(s)\right\}-\boldsymbol{A}\left\{\Phi_{n-k+1}(s) \Omega_{0}(s) Q_{k}^{*}(s)\right\}$. 
If we put (38) into (37) and take into consideration that

$$
\sum_{k=0}^{n} Q_{k}^{*}(s)\left[\Phi_{n-k+1}(s)-\varphi(s) \Phi_{n-k}(s)\right]+Q_{n+1}^{*}(s)=0
$$

for $n=1,2, \ldots$, then we obtain that

$$
\boldsymbol{A}\left\{\varphi(s) \Omega_{n}(s)\right\}=\sum_{k=0}^{n} \Phi_{n-k+1}(s) \boldsymbol{A}\left\{\Omega_{0}(s) Q_{k}^{*}(s)\right\}+\boldsymbol{A}\left\{\Omega_{0}(s) Q_{n+1}^{*}(s)\right\}
$$

for $n=0,1,2, \ldots$ and $\operatorname{Re}(s) \geqslant 0$. By (34) the right-hand side of $(40)$ can be written as $\Omega_{n+1}(s)$ and this proves (36). It remains to show that (39) holds. If we multiply the left-hand side of (39) by $\varrho^{n}$, where $|\varrho|<1$. and add for $n=1,2, \ldots$, then we obtain

$$
\exp \left\{\sum_{k=1}^{\infty} \frac{\varrho^{k}}{k}\left[\varphi_{k}(s)-\varphi_{k}^{+}(s)\right]+\sum_{k=1}^{\infty} \frac{\varrho^{k}}{k}\left[\varphi_{k}^{+}(s)-\varphi_{k}(s)\right]\right\}-\mathbf{1}=0,
$$

whence (39) follows.

6. Further results. First let us define the operator $\boldsymbol{A}$ for a larger class of functions than before. Let us suppose that $\zeta$ is a real or complex random variable for which $\boldsymbol{E}\{|\zeta|\}<\infty$ and let $\eta$ be a real random variable. Write $\eta^{+}=\max (0, \eta)$. The expectation $\boldsymbol{E}\left\{\zeta e^{-8 \eta}\right\}$ exists for $\operatorname{Re}(s)=0$ and determines uniquely $\boldsymbol{E}\left\{\zeta e^{-s \eta^{+}}\right\}$for $\operatorname{Re}(s) \geqslant 0$. Let us write

$$
\boldsymbol{E}\left\{\zeta e^{-s \eta+}\right\}=\boldsymbol{A} \boldsymbol{E}\left\{\zeta e^{-s \eta}\right\} \quad \text { for } \quad \operatorname{Re}(s) \geqslant 0
$$

This definition of $\boldsymbol{A}$ includes the previous one as a particular case.

Theorem 2. If $\operatorname{Re}(s) \geqslant 0$ and $|\varrho|<1$, then we have

$$
\sum_{n=0}^{\infty} \Phi_{n}(s) \varrho^{n}=e^{-A \log [1-\varrho \varphi(s)]}
$$

and

(44) $\quad \sum_{n=0}^{\infty} \Omega_{n}(s) \varrho^{n}=e^{-\boldsymbol{A} \log [1-\varrho \varphi(s)]} \boldsymbol{A}\left\{\Omega_{0}(s) e^{-\log [1-\varrho p(s)]+\boldsymbol{A} \log [1-Q \varphi(s)]}\right\}$,

where $\Phi_{n}(s)(n=0,1,2, \ldots)$ is defined by $(4)$ and $\Omega_{n}(s)(n=0,1,2, \ldots)$ by (33).

Proof. Formula (43) follows from (5) and formula (44) from (34) if we take into consideration that

$$
\sum_{n=1}^{\infty} \frac{\varrho^{n}}{n} \boldsymbol{A}[\varphi(s)]^{n}=\boldsymbol{A}\{-\log [1-\varrho \varphi(s)]\}
$$

for $\operatorname{Re}(s) \geqslant 0$ and $|\varrho|<1$. We can easily prove (45). Suppose that $\xi_{1}, \xi_{2}, \ldots, \xi_{n}, \ldots$ is a sequence of mutually independent random variables and $\boldsymbol{E}\left\{e^{-s \xi_{n}}\right\}=\varphi(s)$ for $n=1,2, \ldots$ and $\operatorname{Re}(s)=0$. Let us suppose that the random variable $\nu$ is independent of $\left\{\xi_{n}\right\}$ and

$$
\boldsymbol{P}\{\boldsymbol{v}=n\}=\frac{1}{n(n+1)}
$$

for $n=1,2, \ldots$ Let us define $\eta=\xi_{1}+\ldots+\xi_{\nu}$ and $\zeta=\varrho^{\nu}(v+1)$. Then

$$
\begin{aligned}
\boldsymbol{E}\{|\zeta|\} & =\sum_{n=1}^{\infty} \frac{|\varrho|^{n}}{n}=-\log (1-|\varrho|)<\infty, \\
\boldsymbol{E}\left\{\zeta e^{-s \eta}\right\} & =\sum_{n=1}^{\infty} \frac{\varrho^{n}}{n}[\varphi(s)]^{n}=-\log (1-\varrho \varphi(s))
\end{aligned}
$$

for $\operatorname{Re}(s) \geqslant 0$ and $|\varrho|<1$ and

$$
\boldsymbol{E}\left\{\zeta e^{-s \eta^{+}}\right\}=\sum_{n=1}^{\infty} \frac{\varrho^{n}}{n} \boldsymbol{A}[\varphi(s)]^{n}
$$

for $\operatorname{Re}(s) \geqslant 0$ and $|\varrho|<1$. Thus (45) follows by (42).

Formulas (43) and (44) are useful because they make it possible to find $\Phi_{n}(s)(n=1,2, \ldots)$ and $\Omega_{n}(s)(n=1,2, \ldots)$ by using the method of factorization.

THEOREM 3. Suppose that $|\varrho|<1$ and that for $\operatorname{Re}(s)=0$

$$
1-\varrho \varphi(s)=\Phi^{+}(s, \varrho) \Phi^{-}(s, \varrho),
$$

where $\Phi^{+}(s, \varrho)$ is a regular function of $s$ in the domain $\operatorname{Re}(s)>0$, continuous and free from zeros in $\operatorname{Re}(s) \geqslant 0$ and satisfies $\lim \log \Phi^{+}(s, \varrho) / s=0$ $(\operatorname{Re}(s) \geqslant 0)$, furthermore $\Phi^{-}(s, \varrho)$ is a regular function of $s$ in the domain $\operatorname{Re}(s)<0$, continuous and free from zeros in $\operatorname{Re}(s) \leqslant 0$ and satisfies $\lim \log \Phi^{-}(s, \varrho) / s=0(\operatorname{Re}(s) \leqslant 0)$. Then we have

$$
\sum_{n=0}^{\infty} \Phi_{n}(s) \varrho^{n}=\frac{1}{\Phi^{+}(s, \varrho) \Phi^{-}(0, \varrho)}
$$

and

$$
\sum_{n=0}^{\infty} \Omega_{n}(s) \varrho^{n}=\frac{1}{\Phi^{+}(s, \varrho)} \boldsymbol{A}\left\{\frac{\Omega_{0}(s)}{\Phi^{-}(s, \varrho)}\right\}
$$

for $\operatorname{Re}(s) \geqslant 0$ and $|\varrho|<1$. 
Proof. Now we have

$$
\boldsymbol{A} \log [1-\varrho \varphi(s)]=\log \Phi^{+}(s, \varrho)+\log \Phi^{-}(0, \varrho)
$$

for $\operatorname{Re}(s) \geqslant 0$ and $|\varrho|<1$. Thus $(51)$ and (52) follow from (43) and (44) respectively.

We can prove (53) for $\operatorname{Re}(s)>0$ if we use the following formula: If $\boldsymbol{E}\{|\zeta|\}<\infty$, then for $\operatorname{Re}(s)>0$ we have

$$
\boldsymbol{E}\left\{\zeta e^{-s \eta^{+}}\right\}=\frac{1}{2} \boldsymbol{E}\{\zeta\}+\frac{s}{2 \pi i} \lim _{e \rightarrow 0} \int_{\dot{L}_{z}} \frac{\boldsymbol{E}\left\{\zeta e^{-z \eta}\right\}}{z(s-z)} d z,
$$

where $L_{\varepsilon}$, the path of integration, consists of the imaginary axis from $z=-i \infty$ to $z=-i \varepsilon$ and again from $z=i \varepsilon$ to $z=i \infty$. By (54) we can obtain (53) for $\operatorname{Re}(s)>0$. Since (53) is continuous for $\operatorname{Re}(s) \geqslant 0$, we can obtain (53) for $\operatorname{Re}(s)=0$ by continuity.

\section{References}

[1] F. Pollaczek, Fonctions caractéristiques de certaines répartitions definies au moyen de la notion d'ordre. Application a la theorie des attentes, C. R. Acad. Sci. 234 (1952), pp. 2334-2336.

[2] F. Spitzer, A combinatorial lemma and its application to probability theory, Trans. Amer. Math. Soc. 82 (1956), pp. 323-339.

\section{Approximation of translation invariant operators \\ by}

DAVID C. SH REVE* (Minnesota)

Abstract. The purpose of this paper is to construct approximations to translation invariant operators from $L^{p}\left(R^{n}\right)$ to $L^{q}\left(R^{n}\right)$. We give error estimates in the form of rates of convergence on subspaces of $L^{p}$.

1. Introduction. The purpose of this paper is to construct a family of approximations $A_{h}, 0<h<\infty$, to a translation invariant operator $A$ from $L^{p}\left(R^{n}\right)$ to $L^{q}\left(R^{n}\right)$. We obtain error estimates

$$
\left\|A u-A_{h} u\right\|_{q} \leqslant C h^{s}\left\|A^{s} u\right\|_{p}
$$

for $u$ in the Bessel potential space $I^{p, s}, s>0$, where $O$ is independent of $h$. For the definition of $\Lambda^{s}$ see Section 4 below.

First we consider $1<p=q<\infty . \quad A_{h}$ is given by $A_{h} u(x)=$ $\sum_{\beta \in Z} c_{\beta, h} u(x+h \beta)$. An interesting feature is that the coefficients $c_{\beta, h}$ are independent of $\dot{h}$ if and only if the multiplier $\hat{T}$ corresponding to $A$ is homogeneous of degree zero, that is, $\hat{T}(\lambda \xi)=\hat{T}(\xi)$ for $\lambda>0$ and $0 \neq \xi \epsilon R^{n}$. We also give approximations to singular integral operators with variable kernels.

In Section 7 we construct approximations $A_{h}$, where $A$ maps $L^{p}$ to $I^{q}, p \leqslant q$. If $p<q$, then $A_{\hbar}$ cannot be a difference operator as above. However, $A_{h} u$ is given by convolving a function with $u$. Certain approximation results for translation invariant operators on locally compact abelian groups are given by Figà-Talamanca and Gaudry [6].

Part of the results presented here appeared in the author's $\mathrm{Ph}$. D. dissertation at Rice University directed by Professor Jim Douglas, Jr.

2. Preliminaries. $R^{n}$ denotes $n$-dimensional Euclidean space, $Z^{n}$ the points in $R^{n}$ with integer coordinates, and $T^{n}$ the dual group of $Z^{n}$. For $r>0$ we set $Q_{r}=\left\{\xi \in R^{n}:-r<\xi_{j} \leqslant r, j=1, \ldots, n\right\}$ and we identify $T^{n}$ with $Q_{\pi} . L^{p}, l^{p}$, and $L^{p}\left(Q_{\pi}\right)$ denote the usual $L^{p}$ spaces of functions on $R^{n}, Z^{n}$, and $Q_{\pi}$ respectively. If $E$ is a subset of $R^{n}, O E$ is the complement of $E$ and $\chi_{E}$ is the characteristic function of $E$.

* During the preparation of this paper the author was partially supported by the National Science Foundation under NSF grant GP-704IX. 